\title{
Endoscopic Treatment of Esophageal Achalasia: Experience of the Hepato-Gastroenterology Service of Fez
}

\author{
Houda Meyiz ${ }^{1,2 *}$, Asmae Lamine ${ }^{1,2}$, Mounia El Yousfi1,2, Nourdin Aqodad ${ }^{1,2}$, \\ Mohammed El Abkari1,2, Adil Ibrahimi1,2, Dafr Allah Benajeh ${ }^{1,2}$, Ihssane Mellouki1,2 \\ ${ }^{1}$ Department of Hepato-Gastroenterology, University Hospital Hassan II, Fez, Morocco \\ ${ }^{2}$ Faculty of Medicine-Fez, University Sidi Mohammed Ben Abdellah, Fez, Morocco \\ Email: ^houdameyiz@hotmail.fr
}

How to cite this paper: Meyiz, H., Lamine, A., El Yousfi, M., Aqodad, N., El Abkari, M., Ibrahimi, A., Benajeh, D.A. and Mellouki, I. (2019) Endoscopic Treatment of Esophageal Achalasia: Experience of the Hepato-Gastroenterology Service of Fez. Open Journal of Gastroenterology, 9, 164-173. https://doi.org/10.4236/ojgas.2019.98019

Received: August 6, 2019

Accepted: August 17, 2019

Published: August 20, 2019

Copyright $\odot 2019$ by author(s) and Scientific Research Publishing Inc. This work is licensed under the Creative Commons Attribution International License (CC BY 4.0).

http://creativecommons.org/licenses/by/4.0/

\begin{abstract}
The achalasia is a rare primary esophageal motor disorder characterized by relaxation disorders of the lower esophageal sphincter and absence of the esophageal body peristalsis. Several studies suggest that the response to the endoscopic treatment depends on several predictors. The aim of our study was to evaluate the endoscopic treatment of esophageal achalasia and identify the predictive factors of endoscopic treatment response. Patients and $\mathrm{Me}$ thods: This is a retrospective analytical study of 78 patients with achalasia, managed in the gastroenterology department of the university medical center Hassan II-Fez, during a period of 5 years (January 2009 to December 2014). The diagnosis of achalasia was retained on a set of clinical, endoscopic, manometric and radiological arguments. A graded dilation protocol starting with a $35 \mathrm{~mm}$ balloon three times for 30 seconds in progressive pressure between 5 and 8 psi was performed. We used the Eckardt score to evaluate the clinical remission. Results: During the study period, 78 patients were included. The average age of our patients was 47 years old [18 - 81] with a sex-ratio M/F of 1.05. The average of Eckardt score before dilation was 5.9 [3 - 9]. An average of 1.41 dilation sessions was performed per patient with $85.9 \%$ of the initial success rate $(n=67)$. Initial success without further dilation sessions was achieved in $55.1 \%$ of our patients $(n=43)$. A clinical recurrence requiring further dilation sessions was observed in $30.8 \%$ of the cases ( $\mathrm{n}$ $=24$ ). The average relapse time after first dilation success was 2.7 years, $75 \%$ occurs within the first year. Dilation failure was retained in 14 patients (17.9\%) requiring surgery. Only one post-dilation perforation was noted. In multivariate analysis, only odynophagia and the number of dilatation sessions were factors of failure of the endoscopic dilation. Conclusion: Pneumatic dilation is a minimally morbid and effective procedure. Our work showed that
\end{abstract}


odynophagia, and the number of dilation sessions, are two predictive factors of endoscopic treatment failure.

\section{Keywords}

Achalasia, Pneumatic Dilatation, Manometry, Eckardt Score

\section{Introduction}

Achalasia is a relatively rare condition with an incidence ranging from 0.3 to 1.63 cases per 100,000 people per year in adults [1] [2] [3]. It is characterized by the absence of peristalsis, incomplete relaxation of the lower esophageal sphincter (LES) with an increased resting tone of LES and, sometimes, increased intraesophageal pressure [4]. Pathologic mechanisms of achalasia remain unknown, although various studies have reported that virus, inflammation, and autoimmune mechanisms may affect the neuronal degeneration of esophageal ganglion cells leading to loss of peristalsis and failure of relaxation of the LES, particularly during swallowing [5] [6]. Since etiology remains unknown, treatment aims, therefore, to relieve symptoms and prevent complications.

Current therapeutic options include pharmacologic therapy, endoscopic treatment, and surgery. The effectiveness of drug treatment is shorter and the recurrence rate is higher [5]. Long term relief can be obtained in about $90 \%$ of cases with either surgical interventions such as laparoscopic Heller myotomy or with endoscopic techniques such as pneumatic dilatation (PD) or, more recently, with per-oral endoscopic myotomy [6]. At present, $\mathrm{PD}$ has proven itself to be the most cost-effective treatment for achalasia over a $5-10$ year period [7] [8]. Several studies suggest that the response to the endoscopic treatment depends on several predictors. The aim of our study was to evaluate endoscopic treatment of esophageal achalasia in the gastroenterology department of the university medical center Hassan II-Fez and identify the predictive factors of endoscopic treatment response.

\section{Material and Methods}

This is a retrospective analytical study of 78 patients with achalasia managed in the gastroenterology department of the university medical center Hassan II-Fez, during a period of 5 years (January 2009 to December 2014).

\subsection{Inclusion Criteria}

o Any patient over 18 years of age, both sexes, with clinical and/or endoscopic and/or manometric +/- radiological signs suggestive of achalasia.

o Absence of other motor disorders.

\subsection{Exclusion Criteria}

o Other known esophageal motor disorders. 


\subsection{Procedure Methodology}

The sources of the various data collected in the patient files were letters from specialist physicians, medical observations in the department, endoscopy, manometry and dilation registry. For each patient, we noted the following data: demographic information, diagnostic procedures, clinical data, paraclinical results, therapeutic management, follow-up, and complications.

All information collected during this work has been treated confidentially. Data collection was retrospective so informed consent was not required.

\subsection{The Technique of Pneumatic Dilatation}

The procedure was carried out by the same work team. We used a Rigiflex Balloon System Figure 1 (Boston Scientific, Marlborough, MA, United States). A graded dilation protocol starting with a $35 \mathrm{~mm}$ balloon three times for 30 seconds in progressive pressure between 5 and 8 psi was performed. The balloon was placed over a guidewire at endoscopy, positioned across the LES and inflated under fluoroscopic guidance. The first dilation is generally realized with a balloon of $35 \mathrm{~mm}$ of the diameter rarely with balloons in $30 \mathrm{~mm}$. we have never used a $40 \mathrm{~mm}$ balloon in our practice. The patients were then kept under observation for 24 hours and can return to normal activities the subsequent day. Further dilation sessions can be performed after a 3 to 4 -week interval if needed on the basis of symptom relief.

\subsection{Clinical Remission}

Clinical symptomatology is evaluated by the symptomatic Eckardt score, composed of four items; dysphagia, chest pain, regurgitation, and weight loss. Each item is scored from 0 to 3 , determining $0=$ no symptoms, $1=$ occasional, $2=$ daily, 3 = at each meal. We studied the evolution of the Eckardt score as a function of time and this at well-defined moments. The first time corresponded to the initial value of the Eckardt score (at the time of diagnosis) and the second time at the end of the first dilation. For patients in remission, a consultation is scheduled after one month and then every six months after the last dilation procedure.

Patients are considered to be in remission if the total symptom score is less than or equal to 3 , or if the item score is less than 2. Failure was defined by lack

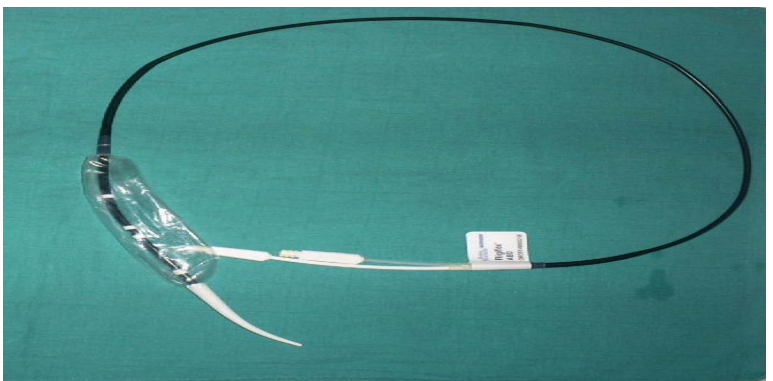

Figure 1. Rigiflex Balloon System (35 mm balloon). 
of improvement, or early recurrence within one month of dilation, or a number of dilation greater than 3 .

The occurrence of gastroesophageal reflux GERD was retained if reflux was previously absent, or esophagitis found at upper endoscopy.

\subsection{Statistical Analysis}

A data Statistical analysis was done using Excel software and Epi Info 2007 for Windows. Initially, a descriptive analysis of the socio-demographic and clinical characteristics as well as a description of the population was performed. Descriptive analysis was performed using proportions calculations for qualitative variables (frequency, percentage), means for quantitative variables. In a second time, the different frequency comparisons were made using the Chi-square test (X2). We proceeded to multivariate analyses by logistic regression. The level $\mathrm{p}<$ 0.05 was considered as the cutoff value or significance.

\section{Results}

During the study period, 78 patients were included. The average age of our patients was 47 years old, with extremes ranging from 18 to 81 years old. The onset of disorders occurred before age 40 for $33 \%$ of our patients $(n=26)$. There was no sex predominance with a sex-ratio $\mathrm{M} / \mathrm{F}$ of 1.05 . The average time from onset of symptoms to diagnosis is 6.8 years with extremes ranging from 1 to 30 years. In terms of clinical presentation, dysphagia was the master symptom found in all our patients $(n=78)$. Weight loss was noted in $85.9 \%$ patients $(n=67)$, regurgitation in $56.4 \%$ of cases $(n=44)$, atypical chest pain in $12.8 \%$ patients $(n=10)$, pyrosis in $16.6 \%$ of cases $(n=13)$, and odynophagia in $20.5 \%$ of cases $(n=16)$. The average of Eckardt score before dilation was 5.9 with extremes ranging from 3 to 9 ('Table 1 ).

Table 1. Clinical characteristics of patients with achalasia, in the gastroenterology department of the university medical center Hassan II-Fez, from January 2009 to December $2014, \mathrm{n}=78$

\begin{tabular}{ccc}
\hline Clinical characteristics & $\mathrm{n}$ & $\%$ \\
\hline Sex-ratio & $1.05(40 \mathrm{M} / 38 \mathrm{~F})$ & \\
Mean age & 47 years $(18-81)$ & \\
Age onset of disorders & & $33.0 \%$ \\
$<40$ years old & 26 & $67.0 \%$ \\
$\geq 40$ years old & 52 & \\
The average time to diagnosis & 6.8 years $(1-30)$ & \\
Clinical presentation & & $100 \%$ \\
Dysphagia & 78 & $85.9 \%$ \\
Weight loss & 67 & $12.8 \%$ \\
Regurgitation & 44 & $16.6 \%$ \\
Chest pain & 10 & $20.5 \%$ \\
pyrosis & 13 & \\
Odynophagia & 16 & \\
Eckardt score before dilation & $5.9(3-9)$ &
\end{tabular}


Esophagogastroduodenoscopy (EGD) was performed for all our patients ( $\mathrm{n}=$ $78)$. The diagnosis of achalasia was suggested in $70.5 \%$ of cases $(n=55)$. Retained food or saliva was found in $60.2 \%$ of cases $(n=47)$, dilated esophagus in $61.5 \%$ of patients $(n=48)$, Absence of peristalsis in $9 \%$ of cases $(n=7)$, and "pop" opening in $56.4 \%$ cases $(\mathrm{n}=44)$. The biopsy was only performed in four patients with esophagitis, it came back normal.

Due to the unavailability, manometry could be performed only in $87.2 \%$ of the patients $(n=68)$. Esophageal aperistalsis was evident in all investigated patients. Incomplete LES relaxation was noted in $55.9 \%$ of patients $(n=38)$. Increased basal LES pressure was observed in $44.1 \%$ of cases $(n=30)$.

A timed barium swallow esophagram (TBA) was performed in 59\% of the patients $(n=46)$. Dilation of the esophagus was assessed in $73.9 \%$ of cases $(n=34)$, a narrow esophagogastric junction (EGJ) with "bird beak" in $43.5 \%$ of cases ( $\mathrm{n}=$ 20 ), and poor emptying of barium in $45.6 \%$ of cases $(n=21)$ (Table 2$)$.

An average of 1.41 dilation sessions was performed per patient with $85.9 \%$ of the initial success rate $(n=67)$. Initial success without further dilation sessions was achieved in $55.1 \%$ of our patients $(n=43)$. A clinical recurrence with further dilation sessions was received in $30.8 \%$ of the cases $(n=24)$ of whom $83.3 \%(n=$ 20) patients required less than 3 sessions with good progression. The average relapse time after first dilation success was 2.7 years, $75 \%$ occurs within the first year. Dilation failure was retained in 14 patients (17.9\%) requiring surgery. Only six cases of immediate complications were noted, 3 cases of GERD, 2 cases of chest pain with fever, and one post-dilation perforation demanding emergency surgery (Table 3 ).

We conducted univariate and multivariate analysis in search of endoscopic treatment predictor's response. Odynophagia $(\mathrm{p}=0.063)$, "pop" opening ( $\mathrm{p}=$ $0.027)$, the number of dilation sessions (more than one dilation session) ( $\mathrm{p}=$

Table 2. Paraclinical results of patients with achalasia, in the gastroenterology department of the university medical center Hassan II-Fez, from January 2009 to December $2014, \mathrm{n}=78$

\begin{tabular}{ccc}
\hline Paraclinical results & $\mathrm{n}$ & $\%$ \\
\hline Esophagogastroduodenoscopy $(\mathbf{n}=\mathbf{7 8})$ & & \\
The diagnosis of achalasia & 55 & $70.5 \%$ \\
Retained food or saliva & 47 & $60.2 \%$ \\
Dilated esophagus & 48 & $61.5 \%$ \\
"pop" opening in cases & 44 & $56.4 \%$ \\
Absence of peristalsis & 7 & $9.0 \%$ \\
Manometry ( $\mathbf{n}=68)$ & & \\
Esophageal aperistalsis & 68 & $100 \%$ \\
Incomplete LES relaxation & 38 & $55.9 \%$ \\
Increased basal LES pressure & 30 & $44.1 \%$ \\
TBA ( $\mathbf{n}=46)$ & & \\
Dilation of the esophagus & 34 & $73.9 \%$ \\
Narrow EGJ & 20 & $43.5 \%$ \\
Poor emptying of barium & 21 & $45.6 \%$ \\
\hline
\end{tabular}


Table 3. Therapeutic management of patients with achalasia, in the gastroenterology department of the university medical center Hassan II-Fez, from January 2009 to December $2014, \mathrm{n}=78$.

\begin{tabular}{ccc}
\hline Therapeutic management & $\mathrm{n}$ & $\%$ \\
\hline Initial success & 67 & $85.9 \%$ \\
Initial success without further dilation & 43 & $55.1 \%$ \\
Dilation number of session & & \\
2 & 20 & $25.6 \%$ \\
3 & 2 & $2.6 \%$ \\
4 & 2 & $2.6 \%$ \\
Dilation failure & 14 & $55.1 \%$ \\
Complications & & \\
GERD & 3 & $3.8 \%$ \\
Chest pain with fever & 2 & $2.6 \%$ \\
Post-dilation perforation & 1 & $1.3 \%$ \\
\hline
\end{tabular}

0.001) were associated with endoscopic treatment failure in univariate study. In multivariate analysis, only odynophagia and the number of dilatation sessions were factors of failure of the endoscopic dilation (Table 4).

\section{Discussion}

Achalasia is a relatively rare condition with an incidence ranging from 0.3 to 1.63 cases per 100,000 people per year in adults [1] [2] [3] [9]. In Morocco, no data are available due to the lack of epidemiological studies. The incidence rate of this pathology seems to be rising [3] [9] [10], it remains unclear if this reflects a true rise in the incidence or an improved diagnosis [6].

Most of the studies found no difference in sex distribution [11] [12] [13] [14]. In our series, there was no sex predominance with a sex-ratio $\mathrm{M} / \mathrm{F}$ at 1.05 , comparable to that noted by Raiss's series [14] and the Iranian series of Yaghoobi [12], where the sex-ratio $\mathrm{M} / \mathrm{F}$ was respectively 1.19 and 1.39 . The average age of our patients is 47.3 years old, it is higher than that found in the Moroccan series of Raiss, where the average age was estimated to 36 years old [14]. Our data are comparable to those reported by some developing countries [11] and slightly lower than in the European ones [15].

In our series, the average time between the onset of clinical signs and diagnosis was prolonged than what found in the other series [11]. This delay may be related to the relative severity of the disease, the patient's adaptation to the symptoms, and inaccessibility to diagnostic means. Dysphagia remains the main symptom in all series and also in our series. The score of Eckardt was at 5.9 in our series comparable to the score noted by Zerbib et al. [15].

Although manometry remains the gold standard for the diagnosis of primitive esophageal achalasia, EGD should be the first examination performed, because it eliminates an organic cause of dysphagia, such as neoplasia or peptic stenosis. Our study showed that EGD is less efficient than esophageal manometry in achalasia. 
Table 4. Risk factors of endoscopic dilation failure of patients achalasia, in the gastroenterology department of the university medical center Hassan II-Fez, from January 2009 to December 2014, $\mathrm{n}=78$.

\begin{tabular}{|c|c|c|c|c|c|c|}
\hline \multirow{2}{*}{\multicolumn{2}{|c|}{ Risk factors }} & \multicolumn{2}{|c|}{ Reccurence } & \multirow{2}{*}{$\mathrm{p}$} & \multirow{2}{*}{ Ajusted OR } & \multirow{2}{*}{ IC95\% } \\
\hline & & Yes & No & & & \\
\hline \multirow{2}{*}{ Age } & $<40 \mathrm{yrs}$ & 8 & 14 & \multirow{2}{*}{0.97} & & \\
\hline & $\geq 40 \mathrm{yrs}$ & 14 & 24 & & & \\
\hline \multirow{2}{*}{ Sex } & Male & 12 & 19 & \multirow{2}{*}{0.73} & & \\
\hline & Female & 10 & 19 & & & \\
\hline \multirow[b]{2}{*}{ Regurgitation } & Yes & 12 & 22 & \multirow[b]{2}{*}{0.80} & & \\
\hline & No & 16 & 10 & & & \\
\hline \multirow{2}{*}{ Odynophagia } & Yes & 12 & 22 & \multirow{2}{*}{0.0036} & 19.19 & \multirow{2}{*}{$1.39-262.8$} \\
\hline & No & 16 & 10 & & 1 & \\
\hline \multirow[b]{2}{*}{ “pop” opening } & Yes & 8 & 25 & \multirow[b]{2}{*}{0.0027} & 1.4 & \multirow[b]{2}{*}{$0.42-56.3$} \\
\hline & No & 14 & 13 & & 1 & \\
\hline \multirow{2}{*}{ Narrow EGJ } & Yes & 14 & 20 & \multirow{2}{*}{0.27} & & \\
\hline & No & 6 & 5 & & & \\
\hline \multirow{2}{*}{$\begin{array}{l}\text { Dilation number of } \\
\text { session }\end{array}$} & 1 & 7 & 28 & \multirow{2}{*}{0.001} & 14.2 & \multirow{2}{*}{$1.54-103.6$} \\
\hline & $>1$ & 14 & 8 & & 1 & \\
\hline
\end{tabular}

Indeed $29.5 \%$ of our patients $(n=23)$, have a normal EGD. These results are similar to the literature data [16]. The National University of Singapore study reported also that $23 \%$ of patients have a normal EDG [16].

A graded dilation protocol starting with a $35 \mathrm{~mm}$ balloon three times for 30 seconds in progressive pressure between 5 and 8 psi was performed in our study. Khan et al. concluded by their prospective randomized study that six seconds is largely sufficient to acquire the effect of PD [17].

PD has proven to be an effective modality for treating achalasia; it allows symptomatic relief while being able to avoid the risks associated with surgery. Pneumatic dilatation with 30,35 and $40 \mathrm{~mm}$ Rigiflex balloons results are good to excellent allowing a symptom relief in $74 \%, 86 \%$ and $90 \%$ of patients respectively at 3years follow up [6]. In our series, using a $35 \mathrm{~mm}$ balloon, the initial success rate is $85.9 \%(n=67)$, and initial success without further dilation sessions was achieved in $55.1 \%$ of our patients $(n=43)$. Mellow was the first to describe the clinical improvement and return of esophageal peristalsis after dilation [18]. In our series, the symptomatic score of Eckardt has been improved after a dilation session with an average passed from 5.91 to 2.18. This improvement of symptoms is more satisfying in Khan's work [17] where the Eckardt score went from 4.2 to 0.78 . This result is logical, given the delay of diagnosis in developing countries which is responsible for a more marked intensity of symptoms.

Up to one-third of patients have complications after PD, most of them are 
minor such as bleeding, fever, chest pain, mucosal esophageal hematoma and mucosal tear without perforation [6]. Perforation is, by far, the most serious complication occurring in about $2.0 \%$ of patients [19]. Indeed, in our series, one post-dilation perforation requiring emergency surgery was noted (1.3\%) [20].

After univariate and multivariate analysis, there was no statistically significant association between sex, age, and recurrence of achalasia symptoms in our study. On the other hand, Ponce et al. showed in a prospective study of 157 patients, that patients under 20 years of age, and male, did not respond well to PD [20]. An association between odynophagia and recurrence of achalasia (Odds Ratio = 19.19; IC95\% [1.39 - 262.8]) was noted in our series. Kostic S et al. reported, whereas, a lack of association between clinical symptoms and recurrence of achalasia [21]. The number of dilations (more than one dilation) is a predictor of endoscopic treatment failure ( $\mathrm{p}=0.001$ ) in our study. Actually, patients who responded to a single PD session had fewer long-term recurrences than those who required more than one session [21].

Several limitations of the study deserve to be cited: the first limitation is the fact that is a retrospective study with long recall periods. The second is the limited number of patients due to the rarity of pathology that may influence the statistical strength of data.

\section{Conclusion}

Primitive achalasia treatment is based on PD or surgery. PD is a simple, minimally morbid and effective procedure. However, risk factors can make this treatment ineffective. Our work showed that odynophagia, and the number of dilation sessions, are two predictive factors of endoscopic treatment failure.

\section{Conflicts of Interest}

The authors declare no conflicts of interest regarding the publication of this paper.

\section{References}

[1] Birgisson, S. and Richter, J.E. (2007) Achalasia in Iceland, 1952-2002: An Epidemiologic Study. Digestive Diseases and Sciences, 52, 1855-1860.

https://doi.org/10.1007/s10620-006-9286-y

[2] Sadowski, D.C., Ackah, F., Jiang, B. and Svenson, L.W. (2010) Achalasia: Incidence, Prevalence and Survival. A Population-Based Study: Achalasia and Epidemiology. Neurogastroenterology \& Motility, 22, e256-e261. https://doi.org/10.1111/j.1365-2982.2010.01511.x

[3] Farrukh, A., DeCaestecker, J. and Mayberry, J.F. (2008) An Epidemiological Study of Achalasia among the South Asian Population of Leicester, 1986-2005. Dysphagia, 23, 161-164. https://doi.org/10.1007/s00455-007-9116-1

[4] Richter, J.E. and Boeckxstaens, G.E. (2011) Management of Achalasia: Surgery or Pneumatic Dilation. Gut, 60, 869-876. https://doi.org/10.1136/gut.2010.212423

[5] Li, H., et al. (2019) The 2 Years' Long-Term Efficacy and Safety of Peroral Endos- 
copic Myotomy for the Treatment of Achalasia: A Systematic Review. Journal of Cardiothoracic Surgery, 14, 1. https://doi.org/10.1186/s13019-018-0811-9

[6] Esposito, D. (2016) Endoscopic Treatment of Esophageal Achalasia. World Journal of Gastrointestinal Endoscopy, 8, 30-39. https://doi.org/10.4253/wjge.v8.i2.30

[7] Karanicolas, P.J., et al. (2007) The Cost of Laparoscopic Myotomy versus Pneumatic Dilatation for Esophageal Achalasia. Surgical Endoscopy, 21, 1198-1206. https://doi.org/10.1007/s00464-007-9364-Z

[8] O’Connor, J.B., Singer, M.E., Imperiale, T.F., Vaezi, M.F. and Richter, J.E. (2002) The Cost-Effectiveness of Treatment Strategies for Achalasia. Digestive Diseases and Sciences, 47, 1516-1525. https://doi.org/10.1023/A:1015811001267

[9] Gennaro, N., et al. (2011) Esophageal Achalasia in the Veneto Region: Epidemiology and Treatment: Epidemiology and Treatment of Achalasia. Journal of Gastrointestinal Surgery, 15, 423-428. https://doi.org/10.1007/s11605-010-1392-7

[10] Howard, P.J., Maher, L., Pryde, A., Cameron, E.W. and Heading, R.C. (1992) Five Year Prospective Study of the Incidence, Clinical Features, and Diagnosis of Achalasia in Edinburgh. Gut, 33, 1011-1015. https://doi.org/10.1136/gut.33.8.1011

[11] Salis, G.B., Mazzadi, S.A., García, A.O. and Chiocca, J.C. (2004) Pneumatic Dilatation in Achalasia of the Esophagus: A Report from Argentina. Diseases of the Esophagus, 17, 124-128. https://doi.org/10.1111/j.1442-2050.2004.00388.x

[12] Yaghoobi, M., Mikaeli, J., Montazeri, G., Nouri, N., Sohrabi, M.R. and Malekzadeh, R. (2003) Correlation between Clinical Severity Score and the Lower Esophageal Sphincter Relaxation Pressure in Idiopathic Achalasia. American Journal of Gastroenterology, 98, 278-283.

[13] Martínek, J., Siroký, M., Plottová, Z., Bures, J., Hep, A. and Spicák, J. (2003) Treatment of Patients with Achalasia with Botulinum Toxin: A Multicenter Prospective Cohort Study. Diseases of the Esophagus, 16, 204-209.

https://doi.org/10.1046/j.1442-2050.2003.00329.x

[14] Raiss, M., Hrora, A., Menfaa, M., Baroudi, S.A., Ahallat, M., Hosni, K., Halhal, A. and Tounsi, A. (2002) L'opération de Heller sans système anti-reflux. À propos de 123 cas. Annales de Chirurgie, 127, 771-775.

https://doi.org/10.1016/S0003-3944(02)00876-3

[15] Zerbib, F., Thétiot, V., Richy, F., Benajah, D.-A., Message, L. and Lamouliatte, H. (2006) Repeated Pneumatic Dilations as Long-Term Maintenance Therapy for Esophageal Achalasia. American Journal of Gastroenterology, 101, 692-697.

[16] Ho, K.-Y., Tay, H.-H. and Kang, J.-Y. (1999) A Prospective Study of the Clinical Features, Manometric Findings, Incidence and Prevalence of Achalasia in Singapore. Journal of Gastroenterology and Hepatology, 14, 791-795. https://doi.org/10.1046/j.1440-1746.1999.01950.x

[17] Khan, A.A., Shah, S.W., Alam, A., Butt, A.K., Shafqat, F. and Castell, D.O. (1998) Pneumatic Balloon Dilation in Achalasia: A Prospective Comparison of Balloon Distention Time. American Journal of Gastroenterology, 93, 1064-1067.

[18] Mellow, M.H. (1976) Return of Esophageal Peristalsis in Idiopathic Achalasia. Gastroenterology, 70, 1148-1151. https://doi.org/10.1016/S0016-5085(76)80329-0

[19] Katzka, D.A. and Castell, D.O. (2011) Review Article: An Analysis of the Efficacy, Perforation Rates and Methods Used in Pneumatic Dilation for Achalasia: Review: Pneumatic Dilation for Achalasia. Alimentary Pharmacology \& Therapeutics, 34, 832-839. https://doi.org/10.1111/j.1365-2036.2011.04816.x

[20] Ponce, J., Garrigues, V., Pertejo, V., Sala, T. and Berenguer, J. (1996) Individual 
Prediction of Response to Pneumatic Dilation in Patients with Achalasia. Digestive Diseases and Sciences, 41, 2135-2141. https://doi.org/10.1007/BF02071392

[21] Kostic, S., et al. (2007) Pneumatic Dilatation or Laparoscopic Cardiomyotomy in the Management of Newly Diagnosed Idiopathic Achalasia. Results of a Randomized Controlled Trial. World Journal of Surgery, 31, 470-478.

https://doi.org/10.1007/s00268-006-0600-9 